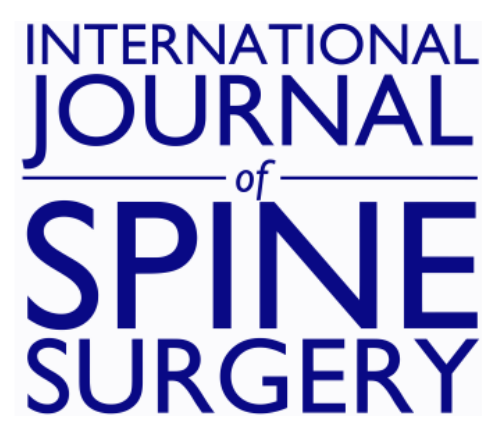

\title{
Case Start Timing of Adult Spinal Deformity Surgeries: Does the Wait Matter?
}

Michael Dinizo, Karan Patel, Igor Dolgalev, Peter G. Passias, Thomas J. Errico and Tina Raman

Int J Spine Surg published online 17 February 2022

http://ijssurgery.com/content/early/2022/03/01/8172

This information is current as of April 26, 2023.

Email Alerts Receive free email-alerts when new articles cite this article. Sign up at: http://ijssurgery.com/alerts 


\title{
Case Start Timing of Adult Spinal Deformity Surgeries: Does the Wait Matter?
}

\author{
MICHAEL DINIZO, MD ${ }^{1} ;$ KARAN PATEL, MD ${ }^{1}$; IGOR DOLGALEV, MS ${ }^{2}$; PETER G. PASSIAS, MD ${ }^{1}$; \\ THOMAS J. ERRICO, $\mathrm{MD}^{3}$; AND TINA RAMAN, MD ${ }^{1}$ \\ ${ }^{I}$ Department of Orthopaedic Surgery, NYU Langone Orthopedic Hospital, New York, NY; ${ }^{2}$ Applied Bioinformatics Laboratory, NYU Langone Medical Center, Miami, \\ FL; ${ }^{3}$ Nicklaus Childrens Hospital, Miami, FL
}

\begin{abstract}
Background: Adult spinal deformity (ASD) surgery can entail complex reconstructive procedures. It is unclear whether there is any effect of case start time on outcomes. We sought to evaluate the effects of case start time and day of the week on 90-day complication, readmission, and revision rates after ASD surgery.

Methods: This is a retrospective study of 1040 ASD patients from a single institution. We collected start times and day of the week for cases from 2011 to 2018. Early start was designated as any case starting either before or at 7:30 AM or between 7:30 and $11 \mathrm{Am}$; late start was designated as any case starting at $11 \mathrm{Am}$ or later. Outcome measures include 90-day complication, revision, and readmission rates.

Results: A total of 1040 ASD patients (age, $46 \pm 23$ years; body mass index, $25 \pm 7$; American Society of Anesthesiologists classification, $2.5 \pm 0.6$; levels fused, $10 \pm 4$; three column osteotomy (3CO), 13\%) were included. There was no association between surgery day of the week and length of stay, 90-day complication, readmission, or reoperation rates in the adjusted analyses. Late start cases had higher rates of 90 -day readmission $(10.5 \%$ vs $6.0 \%, P=0.02)$, reoperation $(11.9 \%$ vs $6.6 \%, P=$ $0.008)$, and neurologic injury $(5.2 \%$ vs $2.1 \%, P=0.019)$. Subanalysis of neurologic complications demonstrated that there was a higher rate of postoperative radiculopathy $(P=0.007)$ and residual central or foraminal stenosis $(P=0.029)$ in late start cases. A late start time was predictive of increased risk for 90 -day readmission $(\mathrm{OR} 1.8, P=0.02)$, unplanned reoperation $(\mathrm{OR} 1.9, P$ $=0.009)$, and neurologic complication (OR 2.1, $P=0.046)$.

Conclusions: A late OR start time was predictive of increased risk for neurologic complication, 90-day readmission, and unplanned reoperation. The well-established protocols for first start OR times for elective ASD surgery may decrease outcome risk and reduce variability in complication rates.

Clinical Relevance: Understanding the impact of start time on outcomes and complications after ASD surgery is helpful for surgeons in preoperative planning and for institutions and hospitals' allocation of operating room staff and resources.

Level of Evidence: 3.

Case Report

Keywords: complex spine surgery, perioperative complications, modifiable risk factor
\end{abstract}

\section{INTRODUCTION}

With the increase in the aging population, degenerative scoliosis and spinal deformity in adults have become growing medical concerns. The prevalence of adult spinal deformity (ASD) increases with each decade of life and has been reported to be between $3 \%$ and $21 \% .{ }^{1,2}$ Coronal and sagittal malalignment, stenosis, and resulting neurologic symptoms that occur over time due to degenerative changes in the spine are a major source of pain and disability in many adults. ${ }^{1,3}$ Patients with ASD have reported a lower quality of life than patients with some chronic medical conditions, such as congestive heart failure and chronic lung disease. ${ }^{3}$ ASD surgery, while complex, can be an effective way to address their malalignment and improve patient function over time. Outcomes after ASD surgery have improved over time, due to developments and innovations in surgical techniques, implants, and available biologics. ${ }^{1,4}$ Despite these improvements, ASD surgeries are complex reconstructive procedures often characterized by osteotomies, long fusion constructs, and long operative times. ${ }^{1,5}$

Spinal deformity surgeries are associated with a high perioperative complication rate. The reported complication rates vary greatly. While some have reported a $8.4 \%$ rate of major complications, others have reported complication rates that range from $13.7 \%$ to $68 \% .^{1,6-9}$ Multiple studies have identified risk factors for complications after ASD surgery. Patient characteristics, such as age, presence of 3 or more medical comorbidities, and severity of spinal deformity have been associated with major perioperative complications after ASD surgery. ${ }^{10,11}$ 
In addition to patient characteristics, surgical variables play a role in their relation to postoperative complications. Significant intraoperative blood loss, particularly blood loss more than 2 or $4 \mathrm{~L}$, can place patients at a higher risk for both major intraoperative and major postoperative complications. ${ }^{9,10}$ Prolonged operative time, particularly if the surgery lasts longer than 7 hours, has been associated with inpatient complications. ${ }^{5}$ The use of multiple approaches has also been identified as a modifiable risk factor for major perioperative complications. ${ }^{6}$

While it is often difficult for surgeons to modify or optimize patient-specific risk factors, such as age and comorbidities, it may be possible to address hospitalbased factors with the goal of reducing postoperative complications. The impact of surgery start time and the day of the week that surgeries are performed have been reported in general surgery, gynecologic surgery, and some orthopedic surgery studies as modifiable factors that can affect postoperative length of stay and postoperative complications. ${ }^{12-18}$ Late surgical start times in orthopedic trauma literature have reported longer surgical times, more blood loss, and more unplanned reoperations, particularly removal of painful hardware, than surgeries that occur earlier in the day. ${ }^{19,20}$ Surgery day of the week has been studied in joint arthroplasty literature. In a retrospective cohort study of 115,053 patients who underwent a primary total knee arthroplasty, surgeries occurring later in the week, on Wednesday, Thursday, or Friday, were found to be associated with increased length of stay and increased costs of admission. $^{21}$

To date, there are no ASD studies that have examined the impact of case start time on postoperative complications. In this regard, the purpose of this study was to evaluate the effects of surgery start time and day of the week on 90-day complication, readmission, and revision rates after ASD surgery.

\section{MATERIALS AND METHODS}

We retrospectively reviewed our institution's prospectively collected database to identify patients who were aged 18 years or older when they underwent posterior spinal fusion for ASD, with a minimum of 4 levels fused, from January 2012 to December 2017. We identified 1040 such patients with complete surgical and inpatient admission data.

\section{Patient and Surgical Characteristics}

The primary indications for surgery were scoliosis or kyphosis that resulted in pain and disability, for which prolonged nonoperative treatment had failed. We used the clinical database and patients' medical records to ascertain patient characteristics including age, sex, and American Society of Anesthesiologists classification. Based on a validated 5-item frailty index, a modified frailty index $(\mathrm{mFI})$ was calculated for each patient, with 5 categories of frailty ranging from 1 (lowest) to 5 (highest). ${ }^{22}$

Surgical parameters collected included number of levels fused, the use of pelvic fixation, interbody fusion, and multirod construct. Each case was assigned a Mirza invasiveness index, based on published criteria, for the purpose of assessing surgical complexity between the 2 groups. ${ }^{23}$

\section{Complications and Unplanned Reoperations Related to Day of Week and Start Time}

We collected start times and day of the week for ASD cases occurring at our institution from 2011 to 2018. Early start was designated as any case starting either before or at 7:30 AM or between 7:30 and $11 \mathrm{Am}$; late start was designated as any case starting at $11 \mathrm{Am}$ and after. Day of week was modeled as a categorical variable, with Monday as the reference group.

Our primary outcomes of interest were complications, unplanned reoperation, and readmission occuring within 90 days of the index surgery. Complications related to technical details of surgery were categorized retrospectively by one attending spine surgeon, the senior author on the study, through review of operative reports and relevant imaging. All clinical information was abstracted from our electronic medical records.

\section{Statistical Methods}

The 2 groups were compared using the $t$ test statistic or Mann-Whitney nonparametric statistic for continuous parameters. In addition, $\chi^{2}$ tests were used for categorical data when all of the expected counts had 5 or more observations, and Fisher exact tests were used for categorical data when any of the expected counts had fewer than 5 observations.

Descriptive statistics were calculated with SPSS version 25 software (IBM, Armonk, NY), R statistical software, and Microsoft Office Excel software (Microsoft Corp., Redmond, Washington). 
Table 1. Characteristics of 1040 adults who underwent surgery for spinal deformity.

\begin{tabular}{|c|c|c|c|c|c|}
\hline \multirow[b]{2}{*}{ Characteristic } & \multicolumn{2}{|c|}{ Early Start $(N=771)$} & \multicolumn{2}{|c|}{ Late Start $(N=269)$} & \multirow[t]{2}{*}{$P$ Value (Early vs Late Start) } \\
\hline & $n(\%)$ & Mean \pm SD & $n(\%)$ & Mean \pm SD & \\
\hline Age, y & & $44 \pm 24$ & & $50 \pm 22$ & 0.10 \\
\hline BMI & & $25 \pm 6$ & & $26 \pm 7$ & 0.52 \\
\hline ASA classification & & $2.4 \pm 0.6$ & & $2.5 \pm 0.6$ & 0.14 \\
\hline $\mathrm{mFI}$ & & $0.4 \pm 0.7$ & & $0.5 \pm 0.7$ & 0.10 \\
\hline Female sex & $454(59)$ & & $183(68)$ & & 0.82 \\
\hline \multicolumn{6}{|l|}{ Comorbidities } \\
\hline Diabetes mellitus & $57(7.4)$ & & $23(8.6)$ & & 0.98 \\
\hline COPD & $29(3.8)$ & & $19(7.1)$ & & 0.09 \\
\hline Cardiovascular disease & $188(24)$ & & $90(34)$ & & 0.10 \\
\hline Osteoporosis & $50(6.5)$ & & $19(7.1)$ & & 0.80 \\
\hline
\end{tabular}

Abbreviations: ASA, American Society of Anesthesiologists; BMI, body mass index; COPD, chronic obstructive pulmonary disease; mFI, modified frailty index.

\section{RESULTS}

\section{Patient Population}

Included in the study were 1040 adults (703 women, $67.8 \%$ ) who underwent surgery for ASD. Among this group, 771 patients $(74.1 \%)$ underwent ASD surgery with an early start time, and 269 (25.9\%) underwent ASD surgery with a late start time. There were 79 revision surgeries, 24 in the late start group and 55 in the early start group $(P=0.68)$.

After controlling for patient and case characteristics, there was no association between day of week and length of stay, 90-day complication, readmission, or reoperation rates in the adjusted analyses.

There were no significant differences found between the early start and late start timing, with regard to preoperative characteristics. There was no difference with respect to age, body mass index (BMI), or frailty score: (first start: age $44 \pm 24$, BMI $25 \pm 6$, mFI $0.4 \pm 0.7$; late start: age: $50 \pm 22$, BMI $26 \pm 7$, $\mathrm{mFI} 0.5 \pm 0.7$ ). There were no differences seen in rate of comorbidities between the groups including diabetes mellitus, chronic obstructive pulmonary disease, congestive heart failure, hypertension, and thromboembolic complications. Full demographic data for the study population are presented in Table 1.

\section{Surgical Characteristics}

In terms of surgical characteristics, early start surgeries had a significantly higher Mirza invasiveness index $(21 \pm 8.2$ vs $19.8 \pm 8.8)$ and significantly greater number of levels fused $(10.4 \pm 4.0$ vs $9.6 \pm 4.4$ levels $)$ than surgeries that were late start $(P<0.05)$. There was no significant difference in percentage of 3-column osteotomy procedures performed in each group. Transforaminal lumbar interbody fusion procedures were done more commonly in late start cases (36\%) vs early start cases $(25 \%)(P=0.04)$. There was no difference in operative time between the 2 groups. There was no difference in length of stay between early start $(6.5 \pm$ 3.8 days) and late start cases $(6.8 \pm 3.9)$. See Table 2 for complete surgical characteristics of the 2 groups.

\section{Complication and Readmission/Revision Rates}

Late start cases had higher rates of 90-day readmission $(11.2 \%$ vs $6.0 \%, P=0.02)$, unplanned reoperation (12.6\% vs $6.6 \%, P=0.008)$, and neurologic injury

Table 2. Surgical characteristics of 1040 adults who underwent surgery for spinal deformity.

\begin{tabular}{|c|c|c|c|c|c|}
\hline \multirow[b]{2}{*}{ Characteristic } & \multicolumn{2}{|c|}{ Early Start $(N=771)$} & \multicolumn{2}{|c|}{ Late Start $(N=269)$} & \multirow[t]{2}{*}{$P$ Value (Early vs Late Start) } \\
\hline & $n(\%)$ & Mean \pm SD & $n(\%)$ & Mean \pm SD & \\
\hline Mirza invasiveness index & & $21 \pm 8.2$ & & $19.8 \pm 8.8$ & 0.04 \\
\hline Number of levels fused & & $10.4 \pm 4.0$ & & $9.6 \pm 4.4$ & 0.01 \\
\hline Operative time (h) & & $6.9 \pm 3.7$ & & $6.5 \pm 3.0$ & 0.12 \\
\hline 3-Column osteotomy & $88(11)$ & & 37 (14) & & 0.85 \\
\hline Posterior column osteotomy & $284(37)$ & & $125(46)$ & & 0.31 \\
\hline TLIF & $192(25)$ & & $96(36)$ & & 0.04 \\
\hline ALIF & $26(3.4)$ & & $8(3.0)$ & & 0.48 \\
\hline Multirod ( $>2$ ) configuration & $451(58)$ & & $167(62)$ & & 0.19 \\
\hline Estimated blood loss (mL) & & $1721 \pm 1596$ & & $1466 \pm 1297$ & 0.16 \\
\hline Length of hospital stay (d) & & $6.5 \pm 3.8$ & & $6.8 \pm 3.9$ & 0.20 \\
\hline
\end{tabular}

Abbreviations: ALIF, anterior lumbar interbody fusion; TLIF, transforaminal lumbar interbody fusion. 
Table 3. Complication and readmission/unplanned reoperation rates in early start vs late start cases.

\begin{tabular}{|c|c|c|c|c|c|}
\hline \multirow[b]{2}{*}{ Parameter } & \multicolumn{2}{|c|}{ Early Start $(N=771)$} & \multicolumn{2}{|c|}{ Late Start $(N=269)$} & \multirow{2}{*}{$\begin{array}{c}P \text { Value } \\
\text { (Early vs Late } \\
\text { Start) }\end{array}$} \\
\hline & $n$ & $\%$ & $n$ & $\%$ & \\
\hline 90-d readmission & 46 & 6.0 & 30 & 11.2 & 0.02 \\
\hline 90-d unplanned reoperation & 51 & 6.6 & 34 & 12.6 & 0.008 \\
\hline \multicolumn{6}{|l|}{ Neurologic complications } \\
\hline Postoperative new onset radiculopathy & 7 & 0.9 & 9 & 3.3 & 0.007 \\
\hline Residual central or foraminal stenosis with persistent radiculopathy & 5 & 0.6 & 6 & 2.2 & 0.029 \\
\hline
\end{tabular}

(5.2\% vs $2.1 \%, 0.019)$. Subgroup analysis of neurologic complications encountered demonstrated that in late start cases, there was a higher rate of postoperative radiculopathy $(3.3 \%$ vs $0.9 \%, P=0.007)$ and residual central or foraminal stenosis $(2.2 \%$ vs $0.6 \%, P=0.029)$ (Table 3).

By regression analysis, late start cases were predictive of increased risk for 90-day readmission (OR 1.8, 95\% CI: $1.1-3.0, P=0.02)$, unplanned reoperation (OR 1.9, 95\% CI: 1.2-3.1, $P=0.009$ ), and neurologic complication (OR 2.1, 95\% CI: 1.0-4.3, $P=0.046$ ) (Table 4).

See Table 5 for a complete list of complications in the 2 groups.

\section{DISCUSSION}

Spinal deformity is an increasingly prevalent and important healthcare problem. Identifying surgical and patient modifiable risk factors may aid in decreasing the rate of perioperative complications in ASD patients. In a series of 1040 patients, we found that a late operative start time, defined as a surgery starting after 11:00 AM, was an independent risk factor for 90-day readmission, unplanned reoperation, and neurologic complications. We found that the rate of neurologic complications in the late start group was significantly higher, specifically the rate of new onset or persistent postoperative radiculopathy, and residual central canal or foraminal stenosis. There were no differences in surgical complexity, operative time, or length of stay between the early start and late start groups. There were no associations identified between the day of the week of surgery and length of stay, 90-day complications, readmissions, or reoperations.

Table 4. Impact of late start time on readmission/unplanned reoperation rates.

\begin{tabular}{lcc}
\hline Parameter & $\boldsymbol{\beta}$ Coefficient (95\% CI) & $\boldsymbol{P}$ Value \\
\hline 90-d readmission & $1.8(1.1-3.0)$ & 0.02 \\
Unplanned reoperation & $1.9(1.2-3.1)$ & 0.009 \\
Neurologic complication & $2.1(1.0-4.3)$ & 0.046 \\
\hline
\end{tabular}

The role of surgical start time on postoperative outcomes has been evaluated in spine surgery. Lang et al conducted a retrospective study where they reported on the outcomes after developing a protocol for outpatient single-level lumbar discectomies. ${ }^{12}$ Although they did not present a time cut-off for a late start, they identified that a later start was responsible for $14 \%$ of their unanticipated same-day admissions, second only to uncontrolled pain (19\%). ${ }^{12}$ Mayo et al found similar results in their 2016 study in which they compared the role of surgery start time on time to discharge for patients undergoing an anterior cervical discectomy and fusion (ACDF). ${ }^{13}$ In their study of 106 patients, the 46 patients with late surgery start times were more likely to require an overnight admission (relative risk $[R R]=1.61$ ) than the 60 patients in the early start cohort because only $26.1 \%$ of late start patients achieved a same-day discharge compared with $45 \%$ of the first start patients. ${ }^{13}$

Other orthopedic studies have investigated the effects of surgery start time. In their prospective, multicenter, nonrandomized study, Ricci et al reported on the effects of surgery start time, particularly daytime vs after hours (starting on or after 4:00 PM), in patients undergoing intramedullary nail fixation of tibial and femoral shaft fractures. ${ }^{19}$ They reported more unplanned reoperations, specifically more frequent removal of painful femoral hardware surgeries needed in their after hours group than in the daytime surgery group. ${ }^{19}$ Similarly, Chacko et al in their retrospective study of start time for hip fracture surgeries reported increased total surgical time and more blood loss in sliding hip screw and intramedullary nail surgeries starting after 6:00 PM when compared with those starting during the day. ${ }^{20}$

Early scheduling is something that can be easily controlled and improved upon, which will potentially reduce complications and readmissions. Early start cases are more likely to be done with an ASD-specific operating room staff and anesthesiologist. Multidisciplinary teams dedicated to spinal deformity have been introduced at multiple institutions and have demonstrated a promising potential toward reducing the burden of complications in patients being treated for 
Table 5. Complications in early start vs late start cases

\begin{tabular}{|c|c|c|c|c|c|}
\hline \multirow[b]{2}{*}{ Parameter } & \multicolumn{2}{|c|}{ Early Start $(N=771)$} & \multicolumn{2}{|c|}{ Late $\operatorname{Start}(N=269)$} & \multirow[b]{2}{*}{$P$ Value } \\
\hline & $n$ & $\%$ & $n$ & $\%$ & \\
\hline Overall number of complications & 71 & 9.2 & 28 & 10.4 & 0.56 \\
\hline Instrumentation-related complication & 13 & 1.7 & 8 & 3.0 & 0.20 \\
\hline Postoperative cerebrospinal fluid leak & 11 & 1.4 & 4 & 1.5 & 0.94 \\
\hline Wound infection & 7 & 0.9 & 6 & 2.2 & 0.09 \\
\hline Seroma & 20 & 2.6 & 10 & 3.7 & 0.34 \\
\hline Pulmonary embolism & 6 & 0.8 & 5 & 1.9 & 0.14 \\
\hline Deep venous thrombosis & 5 & 0.6 & 3 & 1.1 & 0.45 \\
\hline Postoperative pneumonia & 8 & 1.2 & 0 & 0 & 0.09 \\
\hline Ileus & 10 & 1.3 & 4 & 1.5 & 0.82 \\
\hline Postoperative urinary retention & 13 & 1.7 & 1 & 0.4 & 0.11 \\
\hline Sacral fracture & 2 & 0.3 & 0 & 0 & 0.40 \\
\hline Delirium & 5 & 0.6 & 2 & 0.7 & 0.87 \\
\hline Epidural hematoma & 1 & 0.1 & 1 & 0.4 & 0.44 \\
\hline Mortality & 2 & 0.3 & 2 & 0.7 & 0.27 \\
\hline
\end{tabular}

spinal deformities. ${ }^{24}$ Previous studies of patients undergoing ACDF later in the day have shown that there is a higher risk for staying overnight than those patients who have the first surgery of the day, leading to increased resource utilization. ${ }^{13}$ An additional factor that has been well described is the effects of fatigue and decreased resources with late cases, which has been shown to lead to medical errors and decreased staff performance, and cognitive skills. ${ }^{25,26}$

In addition to surgery start time, surgery day of the week has also been studied in orthopedic literature. Khechen et al studied the effect of day of surgery on length of stay and hospital charges after ACDF. In this single institution single-surgeon study, they had 153 patients in the early cohort and 142 patients in the late cohort and they identified no differences between the 2 groups for postoperative complications, hospital length of stay, or hospital costs. ${ }^{14}$ In total joint arthroplasty literature, Pakzad et al studied perioperative risk factors for prolonged hospital stay in 343 patients who underwent a total ankle arthroplasty or ankle arthrodesis and found no association between surgery day of the week and length of stay. ${ }^{27}$ Similarly in their retrospective study on the effects of surgery day of the week on length of stay in 273 total hip arthroplasty (THA) patients, Rathi et al reported that patients who underwent a THA later in the week did not have a longer length of stay than those who were had surgery early in the week (Friday 3.8 days and Monday 3.7 days). ${ }^{28}$ In contrast to these studies, Keswani et al described an association between surgery day of week, surgery start time, and length of stay. ${ }^{15}$ They conducted a retrospective study of 580 THA patients and reported that patients whose surgeries were on Thursday or Friday had a longer length of stay than those whose surgeries were on Monday or Tuesday (3.7 vs 3.4 days). ${ }^{15}$ Furthermore, patients whose surgery start time was after 2 PM had a longer hospital length of stay than patients who were operated on before 2 PM (3.9 vs 3.5 days). ${ }^{15}$ The association between total joint arthroplasty surgery later in the week and extended length of stay when compared to surgeries early in the week has also been reported by Muppavarapu et al and Newman et al. ${ }^{29,30}$ Newman et al also reported that performing a total knee arthroplasty later in the week was further associated with higher hospital costs than a total knee arthroplasty performed early in the week $(\$ 44,175$ vs $\$ 42,772)^{30}$

Contrary to other studies, this present study did not find an association with surgery start time or surgery day of the week and length of stay. The increased risk of perioperative complications, readmissions, and reoperations with a late surgery start time is a novel and important finding. Decreasing such complications after ASD surgery is necessary, given the rise in the prevalence of ASD and increase in number of surgeries performed for this pathology. Surgery start time is a factor that can easily be modified, and protocols can be established to ensure first start times for ASD surgery to potentially decrease complication rates.

The present study has both strengths and weaknesses. As this investigation involved the use of our institution's prospectively collected database, we were able to identify and review well-compiled data for the 1040 patients included in this study. Our data set was unable to capture the reason for late start, whether it was scheduled or due to OR delays. Future studies may also evaluate the difference between first-half of the week (Monday-Wednesday) vs end of the week surger

y (Thursday-Friday), which may give additional information in order to guide other surgeons. The retrospective nature of this study, however, may introduce elements of reporting and selection bias. Other 
limitations of this study include lack of generalizability given it is single institution analysis and lack of radiological data on severity of pathology/deformity.

Future investigations on the effect of surgery start time and surgery day of the week are necessary to further characterize the risks associated with these factors.

\section{CONCLUSION}

A late start time for ASD surgeries is a risk factor for 90-day readmission, unplanned reoperations, and neurologic complications. Establishing well-designed protocols that prioritize an early start time for ASD surgeries may help decrease the risks and complication rates associated with these procedures.

\section{REFERENCES}

1. Soroceanu A, Burton DC, Oren JH, et al. Medical complications after adult spinal deformity surgery: incidence, risk factors, and clinical impact. Spine. 2016;41(22):1718-1723. doi:10.1097/ BRS.0000000000001636

2. Kebaish KM, Neubauer PR, Voros GD, Khoshnevisan MA, Skolasky RL. Scoliosis in adults aged forty years and older: prevalence and relationship to age, race, and gender. Spine. 2011;36(9):731-736. doi:10.1097/BRS.0b013e3181e9f120

3. Pellisé F, Vila-Casademunt A, Ferrer M, et al. Impact on health related quality of life of adult spinal deformity (ASD) compared with other chronic conditions. Eur Spine J. 2015;24(1):3-11. doi:10.1007/s00586-014-3542-1

4. Bess S, Line BG, Lafage $V$, et al. Does recombinant human bone morphogenetic protein-2 use in adult spinal deformity increase complications and are complications associated with location of rhBMP-2 use? A prospective, multicenter study of 279 consecutive patients. Spine. 2014;39(3):233-242. doi:10.1097/ BRS.0000000000000104

5. Samuel AM, MC F, Anandasivam NS, et al. After posterior fusions for adult spinal deformity, operative time is more predictive of perioperative morbidity, rather than surgical invasiveness: a need for speed? Spine. 2017;42(24):1880-1887. doi:10.1097/ BRS.0000000000002243

6. Schwab FJ, Hawkinson N, Lafage V, et al. Risk factors for major peri-operative complications in adult spinal deformity surgery: a multi-center review of 953 consecutive patients. Eur Spine J. 2012;21(12):2603-2610. doi:10.1007/s00586-012-2370-4

7. Charosky S, Guigui P, Blamoutier A, Roussouly P, Chopin D, Study Group on Scoliosis. Complications and risk factors of primary adult scoliosis surgery: a multicenter study of 306 patients. Spine. 2012;37(8):693-700. doi:10.1097/BRS.0b013e31822ff5c1

8. Cho SK, Bridwell KH, Lenke LG, et al. Major complications in revision adult deformity surgery: risk factors and clinical outcomes with 2- to 7-year follow-up. Spine. 2012;37(6):489-500. doi:10.1097/BRS.0b013e3182217ab5

9. Cho K-J, Suk S-I, Park S-R, et al. Complications in posterior fusion and instrumentation for degenerative lumbar scoliosis. Spine. 2007;32(20):2232-2237. doi:10.1097/BRS.0b013e31814b2d3c

10. Bianco $\mathrm{K}$, Norton $\mathrm{R}$, Schwab F, et al. Complications and intercenter variability of three-column osteotomies for spinal deformity surgery: a retrospective review of 423 patients. Neurosurg Focus. 2014;36(5):1-7. doi:10.3171/2014.2.FOCUS1422

11. Auerbach JD, Lenke LG, Bridwell KH, et al. Major complications and comparison between 3-column osteotomy techniques in 105 consecutive spinal deformity procedures. Spine. 2012;37(14):1198-1210. doi:10.1097/BRS.0b013e31824fffde

12. Lang S-S, Chen HI, Koch MJ, et al. Development of an outpatient protocol for lumbar discectomy: our institutional experience. World Neurosurg. 2014;82(5):897-901. doi:10.1016/j. wneu.2014.05.035

13. Mayo BC, MasselDH, Bohl DD, Long WW, Modi KD, Singh K. Effect of surgery start time on day of discharge in anterior cervical discectomy and fusion patients. Spine. 2016;41(24):1939-1944. doi:10.1097/BRS.0000000000001627

14. Khechen B, Haws BE, Patel DV, et al. Does the day of the week affect length of stay and hospital charges following anterior cervical discectomy and fusion? Int J Spine Surg. 2019;13(3):296-301. doi: $10.14444 / 6040$

15. Keswani A, Beck C, Meier KM, Fields A, Bronson MJ, Moucha CS. Day of surgery and surgical start time affect hospital length of stay after total Hip arthroplasty. J Arthroplasty. 2016;31(11):2426-2431. doi:10.1016/j.arth.2016.04.013.

16. Akoh JA, Watson WA, Bourne TP. Day case laparoscopic cholecystectomy: reducing the admission rate. Int J Surg. 2011;9(1):63-67. doi:10.1016/j.ijsu.2010.09.002

17. Robinson TN, Biffl WL, Moore EE, Heimbach JK, Calkins CM, Burch JM, et al. Predicting failure of outpatient laparoscopic cholecystectomy. Am J Surg. 2002;184(6):515-518. doi:10.1016/ s0002-9610(02)01080-2

18. Gien LT, Kupets R, Covens A. Feasibility of sameday discharge after laparoscopic surgery in gynecologic oncology. Gynecol Oncol. 2011;121(2):339-343. doi:10.1016/j. ygyno.2010.12.344

19. Ricci WM, Gallagher B, Brandt A, Schwappach J, Tucker M, Leighton R. Is after-hours orthopaedic surgery associated with adverse outcomes? A prospective comparative study. J Bone Joint Surg Am. 2009;91(9):2067-2072. doi:10.2106/JBJS.H.00661

20. Chacko AT, Ramirez MA, Ramappa AJ, Richardson LC, Appleton PT, Rodriguez EK. Does late night hip surgery affect outcome? J Trauma. 2011;71(2):447-453. doi:10.1097/ TA.0b013e3182231ad7

21. Boylan MR, Perfetti DC, Naziri Q, Maheshwari AV, Paulino CB, Mont MA. Is day of surgery associated with adverse clinical and economic outcomes following primary total knee arthroplasty? J Arthroplasty. 2017;32(8):2339-2346. doi:10.1016/j. arth.2017.03.029

22. Subramaniam S, Aalberg JJ, Soriano RP, Divino CM. New 5-factor modified frailty index using American college of surgeons NSQIP data. J Am Coll Surg. 2018;226(2):173-181. doi:10.1016/j. jamcollsurg.2017.11.005.

23. Mirza SK, Deyo RA, Heagerty PJ, et al. Development of an index to characterize the "invasiveness" of spine surgery: validation by comparison to blood loss and operative time. Spine. 2008;33(24):2651-2661. doi:10.1097/BRS.0b013e31818dad07

24. Friedman GN, Benton JA, Echt M, et al. Multidisciplinary approaches to complication reduction in complex spine surgery: a systematic review. Spine J. 2020;20(8):1248-1260. doi:10.1016/j. spinee.2020.04.008.

25. Nurok M, Gewertz BL. Outcomes of procedures performed by attending surgeons after night work. $N$ Engl J Med. 2015;373(24):2384. doi:10.1056/NEJMc1512756. 
26. Sinha A, Singh A, Tewari A. The fatigued anesthesiologist: a threat to patient safety? J Anaesthesiol Clin Pharmacol. 2013;29(2):151-159. doi:10.4103/0970-9185.111657

27. Pakzad H, Thevendran G, Penner MJ, Qian H, Younger A. Factors associated with longer length of hospital stay after primary elective ankle surgery for end-stage ankle arthritis. J Bone Joint Surg Am. 2014;96(1):32-39. doi:10.2106/JBJS.K.00834

28. Rathi P, Coleman S, Durbin-Johnson B, Giordani M, Pereira G, Di Cesare PE. Effect of day of the week of primary total hip arthroplasty on length of stay at a university-based teaching medical center. Am J Orthop. 2014;43(12):E299-E303

29. Muppavarapu RC, Chaurasia AR, Schwarzkopf R, Matzkin EG, Cassidy CC, Smith EL. Total joint arthroplasty surgery: does day of surgery matter? J Arthroplasty. 2014;29(10):1943-1945. doi:10.1016/j.arth.2014.06.004.

30. Newman JM, Szubski CR, Barsoum WK, Higuera CA, Molloy RM, Murray TG. Day of surgery affects length of stay and charges in primary total Hip and knee arthroplasty. J Arthroplasty. 2017;32(1):11-15. doi:10.1016/j.arth.2016.06.032

Funding: The author(s) received no financial support for the research, authorship, and/or publication of this article.
Declaration of Conflicting Interests: Peter G. Passias reports personal fees from Globus Medical, Medicrea, Royal Biologics, SpineWave, Terumo, and Zimmer Biomet; and other from Allosource and the Cervical Scoliosis Research Society (cSRS) outside of the submitted work. Thomas J. Errico reports personal fees from Stryker and K2M outside of the submitted work. The remaining authors have nothing to report.

Corresponding Author: Tina Raman, NYU Langone Orthopedic Hospital, 171 Delancey Street, New York, NY 10003, USA; tina.raman@ nyulangone. org

Published 16 February 2022

This manuscript is generously published free of charge by ISASS, the International Society for the Advancement of Spine Surgery. Copyright (C) 2022 ISASS. To see more or order reprints or permissions, see http:// ijssurgery.com. 